\title{
Intrinsic Quantum Correlations of Weak Coherent States for Quantum Communication.
}

Yong Meng Sua, Erin Scanlon, Travis Beaulieu, Viktor Bollen, and Kim Fook Lee ${ }^{1, *}$

\author{
${ }^{1}$ Department of Physics, \\ Michigan Technological University, \\ Houghton, Michigan 49931
}

(Dated: October 22, 2018)

\begin{abstract}
Intrinsic quantum correlations of weak coherent states are observed between two parties through a novel detection scheme, which can be used as a supplement to the existence decoy-state BB84 and differential phase-shift quantum key distribution (DPS-QKD) protocols. In a proof-of-principle experiment, we generate bi-partite correlations of weak coherent states using weak local oscillator fields in two spatially separated balanced homodyne detections. We employ nonlinearity of postmeasurement method to obtain the bi-partite correlations from two single-field interferences at individual homodyne measurement. This scheme is then used to demonstrate bits correlations between two parties over a distance of $10 \mathrm{~km}$ through a transmission fiber. We believe that the scheme can add another physical layer of security to these protocols for quantum key distribution.
\end{abstract}


Quantum entanglement and superposition provide secure communication between two parties for key generation and information processing. However, entanglement based key generation such as Ekert's protocol [1] is hard to implement in real-world optical fiber network because bi-partite correlations of entangled photon-pairs are sensitive to loss. There has been much interest in quantum key generation of using weak coherent states or highly attenuated lasers. Quantum key distribution using weak coherent states, such as coherent state differential phase-shift quantum key distribution (DPS-QKD) [2, 3] and decoy-states BB84 protocols [4 9], have been proven to be unconditional secure against photon-number splitting attack (PNS). The DPS-QKD uses intrinsic correlations between the relative phase shifts $\{0, \pi\}$ of two consecutive pulses to achieve unconditional security between two parties by constructing equivalent states for the entanglement-based protocol [3]. The decoy state quantum key distribution uses intrinsic correlations between the relative mean photon numbers of two set of weak coherent states to detect PNS attack in BB84 protocol [4]. Meanwhile, Y00 protocol [10] uses intrinsic correlations between phase and mean photon number fluctuations of weak coherent states to provide cryptographic service of data encryption between two parties.

Intrinsic quantum correlations of coherent states can be prepared, measured and shared between two parties for quantum cryptography. We are motivated to propose a scheme based on weak coherent states for generating intrinsic bi-partite correlations as a supplement resource to the existence protocols such as coherent state DPS-QKD and decoy state BB84.

Weak local oscillator (LO) field in a coherent state has been successfully used to directly measure bi-partite correlation functions of a two-photon source and violate Bell's inequalities using homodyne detection with photon counting [11]. In this work, we employ a weak local oscillator field in a coherent state to extract intrinsic correlations of weak coherent states between two parties using balanced homodyne measurement. Briefly, we first prepare a weak coherent state using a highly attenuated laser at telecom wavelength. The coherent state is split by a 50/50 beam splitter and sent to Alice and Bob. Alice and Bob, each has a balanced homodyne detection scheme for measuring his/her coherent state with a weak local oscillator field. We employ nonlinearity of post-measurement method, i.e., multiply two single-field interferences from individual balanced homodyne measurement. Then, the mean-value of the multiplied signal provides raw data of field-field correlations of weak coherent states. We normalize the raw data with the mean photon numbers of weak coherent state and LO 


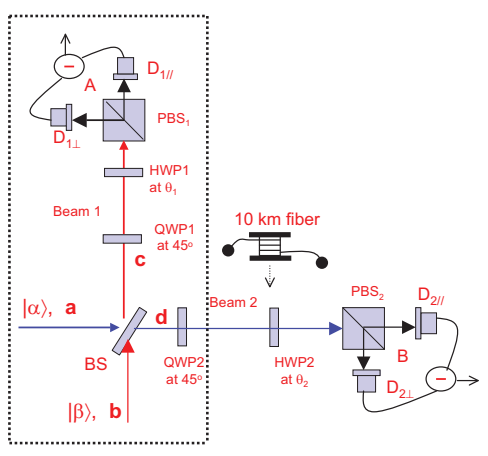

FIG. 1: Experiment setup for observing coherent state bi-partite correlation of weak coherent states.

field to obtain the coherent state bi-partite correlation function (CSBC). It is named so as to avoid confusion with bi-partite correlations provided by four Bells states of polarizationentangled photon pair. Four types of correlation functions $\pm \cos 2\left(\theta_{1} \pm \theta_{2}\right)$ can be prepared by using linear optics devices in Alice or Bob alone, where $\theta_{1}$ and $\theta_{2}$ are the projection angles of the analyzers at Alice and Bob. This means that Alice can keep her copy of the coherent state and send another copy to Bob. By locally changing the relative phases between her coherent state and weak local oscillator field, her acts will change the correlation functions shared with Bob. Once we establish one of the four correlation functions between Alice and Bob over a distance of $10 \mathrm{~km}$ through a transmission fiber, we change the phases of the weak local oscillator field $\{0, \pi\}$ for implementing bits correlations between them.

The weak coherent state $|\alpha\rangle$ and weak LO field in a coherent state $|\beta\rangle$ are treated as a product state of two independent coherent states $|\alpha, \beta\rangle$ in the input of the beam splitter [12]. The density matrix of the output state from the beam splitter is depended on the integration over the phase space of P-representation for the input product state. Since the $|\alpha\rangle$ and $|\beta\rangle$ are intrinsically correlated from the same laser through the LO phases $\{0,2 \pi\}$, then integration over the phase-spaces of $\alpha$ and $\beta$ can produce the output state that is intrinsically entangled [13]. This is accomplished in our experiment by conducting the mean-value measurement of the multiplied signals of the output state. The two mode coherent states at the output of the beam splitter, $|\alpha+\beta\rangle_{1}|\alpha-\beta\rangle_{2}$, can be manipulated by linear phase shifters to project out coherent interferences parts $\left|\alpha_{1} \beta_{2}\right\rangle \pm\left|\beta_{1} \alpha_{2}\right\rangle$ or $\left|\alpha_{1} \alpha_{2}\right\rangle \pm\left|\beta_{1} \beta_{2}\right\rangle$ plus phase-space noises of $|\alpha\rangle$ and $|\beta\rangle$.

A proof-of-principle experiment for the brief description above is shown in Fig.1. A 
highly attenuated laser at telecom-band wavelength of $1534 \mathrm{~nm}$ is used to provide weak coherent states and weak local oscillator field. We use a 50/50 beam splitter (BS) to mix the horizontally polarized coherent state $|\alpha\rangle$ and the vertically polarized local oscillator field $|\beta\rangle$. The input field operators $\hat{a}$ and $\hat{b}$ at the beam splitter are the annihilation operators for the coherent state $|\alpha\rangle$ and the LO field $|\beta\rangle$, respectively. Using unitary transformation matrix of a 50/50 beam splitter, the output modes at the beam splitter are $\hat{c_{1}}=\frac{1}{\sqrt{2}}(\hat{a} \mathbf{x})+i \hat{b} \mathbf{y}$ in beam 1 and $\hat{d}_{2}=\frac{1}{\sqrt{2}}(i \hat{a} \mathbf{x})+\hat{b} \mathbf{y}$ in beam 2. A quarter wave plate (QWP) at $45^{\circ}$ is inserted at beam 1 and beam 2 to transform the linearly polarized states to circularly polarized states. Then, after the matrix transformation of a quarter wave plate, the field operators $\hat{c_{1}} \rightarrow{\hat{c_{1}}}^{\prime}=\frac{1}{\sqrt{2}}((\hat{a}+\hat{b}) \mathbf{x}+i(\hat{b}-\hat{a}) \mathbf{y})$ and $\hat{d}_{1} \rightarrow \hat{d}_{1}^{\prime}=\frac{1}{\sqrt{2}}((i \hat{a}-\hat{b}) \mathbf{x}+(\hat{b}+\hat{a}) \mathbf{y})$. A half-wave plate HWP1 (HWP2) is inserted in beam 1 (2) before a cube polarization beam splitter PBS1 (PBS2) to project out the polarization state $\theta_{1}\left(\theta_{2}\right)$ with unit vector $\hat{e_{1(2)}}=\cos \theta_{1(2)} \mathbf{x}+\sin \theta_{1(2)} \mathbf{y}$, respectively. The field operators ${\hat{c_{1}}}^{\prime \prime}=\left(\hat{c_{1}} \cdot \hat{e_{1}}\right) \hat{e_{1}}$ after the PBS1 at beam 1 and $\hat{d}_{2}{ }^{\prime \prime}=\left({\hat{d_{2}}}^{\prime} \cdot \hat{e_{2}}\right) \hat{e_{2}}$ after the PBS2 at beam 2 provide the photon number operators of the transmitted component of the combined fields as,

$$
\begin{aligned}
\hat{c}_{1}^{* \prime} \hat{c}_{1}^{\prime \prime} & =\frac{1}{2}\left[a^{*} \hat{a}+\hat{b^{*}} \hat{b}+\hat{a^{*}} \hat{b} e^{i 2 \theta_{1}}+\hat{b^{*}} \hat{a} e^{-i 2 \theta_{1}}\right] \\
\hat{d}_{2}^{* \prime \prime} \hat{d}_{2}^{\prime \prime} & =\frac{1}{2}\left[a^{*} \hat{a}+\hat{b^{*}} \hat{b}-\hat{a^{*}} \hat{b} e^{i 2 \theta_{2}}-\hat{b^{*}} \hat{a} e^{-i 2 \theta_{2}}\right] .
\end{aligned}
$$

Now, using the input weak coherent state $|\alpha\rangle=\left|\alpha e^{i \phi_{\alpha}}\right\rangle$ and the input weak LO field in a coherent state $|\beta\rangle=\left|\beta e^{i \phi_{L O}}\right\rangle$ into Eq. 11 and Eq. 2, the detectors A and B measure the transmitted components of the beat intensities,

$$
\begin{aligned}
& I_{1 \|}\left(\theta_{1}, \phi_{L O}\right) \rightarrow\left\langle\alpha, \beta\left|\hat{c}^{*}{ }^{\prime \prime} \hat{c}^{\prime \prime}\right| \alpha, \beta\right\rangle=\eta_{A}\left[|\alpha|^{2}+|\beta|^{2}+|\alpha||\beta| \operatorname{Cos}\left(2 \theta_{1}+\phi_{L O}-\phi_{\alpha}\right)\right] \\
& I_{2 \|}\left(\theta_{2}, \phi_{L O}\right) \rightarrow\left\langle\alpha, \beta\left|{\hat{d^{*}}}^{\prime \prime} \hat{d}^{\prime \prime}\right| \alpha, \beta\right\rangle=\eta_{B}\left[|\alpha|^{2}+|\beta|^{2}-|\alpha||\beta| \operatorname{Cos}\left(2 \theta_{2}+\phi_{L O}-\phi_{\alpha}\right)\right]
\end{aligned}
$$

where $|\alpha, \beta\rangle$ is the input state of the beam splitter and $\eta_{A}\left(\eta_{B}\right)$ is the conversion efficiency (Watt $\rightarrow$ Current) for detection electronics of A (B). The first two terms are intensities of the two coherent states and the last term is the interference term consists of polarization angle $\theta_{1}\left(\theta_{2}\right)$, the phases of LO $\left(\phi_{L O}\right)$ and the weak coherent state $\left(\phi_{\alpha}\right)$. The beat intensity $I_{1 \|}\left(\theta_{1}, \phi_{L O}\right)$ is anti-correlated to $I_{2 \|}\left(\theta_{2}, \phi_{L O}\right)$ because of the $\pi$-phase shift induced by the 50/50 beam splitter. The beat intensity for the reflected signal at the PBS1 (PBS2)is 
$I_{1 \perp}\left(\theta_{1 \perp}, \phi_{L O}\right)\left(I_{2 \perp}\left(\theta_{2 \perp}, \phi_{L O}\right)\right)$, where the $\theta_{1 \perp}=\theta_{1}+\pi / 2\left(\theta_{2 \perp}=\theta_{2}+\pi / 2\right)$, respectively. Then, the detectors $\mathrm{A}$ and $\mathrm{B}$ measure the balanced homodyne beat intensities, that are,

$$
\begin{gathered}
\mathcal{A}_{1} \rightarrow I_{1 \|}-I_{1 \perp}=2 \eta_{A}|\alpha||\beta| \cos \left(2 \theta_{1}+\phi_{L O}-\phi_{\alpha}\right) \\
\mathcal{B}_{2} \rightarrow I_{2 \|}-I_{2 \perp}=-2 \eta_{B}|\alpha||\beta| \cos \left(2 \theta_{2}+\phi_{L O}-\phi_{\alpha}\right)
\end{gathered}
$$

plus shot noise at each detection of $\mathcal{A}_{1}$ and $\mathcal{B}_{2}$. The individual intensities of the two coherent states are subtracted. One can see that the information $2 \theta_{1}+\phi_{L O}$ and $2 \theta_{2}+\phi_{L O}$ are protected by quantum phase noise $\phi_{\alpha}$ with phase fluctuation of $\Delta \phi_{\alpha} \geq \frac{1}{\Delta n}$, where low mean photon number fluctuation associated with phase fluctuation is provided by the weak coherent state. The balanced homodyne beat intensities in detectors A and B are then multiplied to obtain,

$$
\mathcal{A}_{1} \mathcal{B}_{2}=-2 \eta_{A} \eta_{B}|\alpha|^{2}|\beta|^{2}\left[\cos 2\left(\theta_{1}-\theta_{2}\right)+\cos 2\left(\theta_{1}+\theta_{2}+\phi_{L O}-\phi_{\alpha}\right)\right] .
$$

The multiplied balanced-homodyne beat intensities did not provide bi-partite correlation function directly. This is predicted because so far we have performed the multiplication of single-field interferences obtained from individual balanced homodyne detection at the detectors A and B. However, by taking the mean value of this multiplied beat intensities, the last term is averaged to zero due to slowly varying local oscillator phase $\phi_{L O}$ from $\{0,2 \pi\}$ protected by randomness of quantum phase noise $\phi_{\alpha}$. Note that $\Delta \phi_{\alpha}$ cannot randomly provide phase shift $0 \rightarrow 2 \pi$. And hence, we obtain the expectation value of two detectors or coherent state bi-partite correlation function as given by,

$$
\overline{\mathcal{A}_{1} \mathcal{B}_{2}} \rightarrow\left\langle\mathcal{A}_{1} \mathcal{B}_{2}\right\rangle=-2 \eta_{A} \eta_{B}|\alpha|^{2}|\beta|^{2} \cos 2\left(\theta_{1}-\theta_{2}\right)
$$

The coherent state bi-partite correlation is protected by the term $\cos 2\left(\theta_{1}+\theta_{2}+\phi_{L O}-\phi_{\alpha}\right)$, which is averaged to zero. In real practice, the product of mean photon numbers $|\alpha|^{2}|\beta|^{2}$ is obtained by setting the correlation function to its maximum obtainable value, that is, $\theta_{1}=\theta_{2}$. The raw data of the multiplied beat intensities is then normalized with the product of $2 \eta_{A} \eta_{B}|\alpha|^{2}|\beta|^{2}$ to obtain correlation function $-\cos 2\left(\theta_{1}-\theta_{2}\right)$. As for the use of quantum communication between two distant observers, we have to establish the bi-partite correlation function and then to implement bits correlations at detectors A and B through the LO phases $\{0, \pi\}$ without using the post-measurement method. To verify the analysis above, we perform systematic studies of the experiment. The balanced homodyne detectors are made of two PIN photodiodes (EXT500). We use a piezoelectric transducer (PZT) to ramp the 

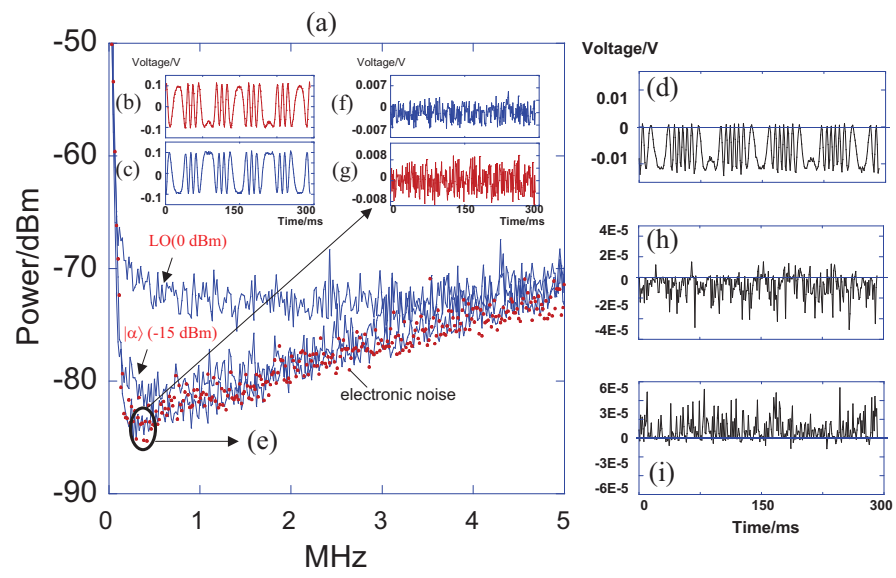

FIG. 2: (a) Shot noises of the weak LO field $(0 \mathrm{dBm})$ and coherent state $(-15 \mathrm{dBm})$, and the corresponding beat signals for $\theta_{1}=\theta_{2}$ at detector A (b) and detector B (c), and their multiplied signal (d). (e) shot noises for the weak LO field $(-30 \mathrm{dBm})$ and the corresponding beat signals for $\theta_{1}=\theta_{2}$ at detector A (f) and detector B (g), and their multiplied beat signal (h). (i) the multiplied beat signal when the relative angle $\theta_{1}-\theta_{2}=\pi / 2$. (square dot) Electronic noise.

phase of the weak LO beam. We first perform the experiment by using a strong LO field and a coherent state with average power of $0 \mathrm{dBm}$ and $-15 \mathrm{dBm}$, respectively. Figure 2 (a) shows the spectrum of the shot noise levels of the strong LO field and coherent state, and the electronic noise of our detection system. We set the relative angle between Alice's and Bob's analyzers as $\theta_{1}-\theta_{2}=0$. The beat intensities at detectors $\mathrm{A}$ and $\mathrm{B}$ are shown in Fig. 2(b) and (c)(inset of Fig.2(a)). The discontinuity of the beats are due to the ramping of the PZT. With these large mean photon fluxes, the interference signals are stable as predicted by the coherent states with large mean photon number. The product of the beat intensities is shown in Fig.2(d), which is a clear indication of anti-correlation between single-field interferences at detectors $\mathrm{A}$ and $\mathrm{B}$. Then, we attenuate the laser light to obtain weak LO field and weak coherent state with average power of $-30 \mathrm{dBm}$ and $-30 \mathrm{dBm}$, respectively. All the average optical powers reported in this work are measured just before the PBS1(PBS2). Figure 2(e) shows the shot noise of weak LO field almost falls on the electronic noise spectrum. We observe the beat intensities at detectors A and B as shown in Fig. 2(f) and (g) (inset of Fig.2(a)) with the interference signals hidden or protected by the shot noises of the LO field, quantum phase noise $\phi_{\alpha}$ of the weak coherent state due to low mean photon number fluctuation, and electronic noises. These are predicted by the Eq. 5 and Eq. 6. In 
the experiment, the beat intensities at detectors $\mathrm{A}$ and $\mathrm{B}$ are stored in their computers. Then, these raw data are multiplied together as shown in Fig. 2(h). The multiplied beat intensity consists of two parts; coherent and noise interferences. The coherent interference part contains the term $-2 \eta_{A} \eta_{B}|\alpha|^{2}|\beta|^{2} \cos 2\left(\theta_{1}-\theta_{2}\right)$. The noise interference part contains the term $-2 \eta_{A} \eta_{B}|\alpha|^{2}|\beta|^{2} \cos 2\left(\theta_{1}+\theta_{2}+\phi_{L O}-\phi_{\alpha}\right)$ which is averaged to zero because of the periodic of the LO phase, $\phi_{L O}=\{0,2 \pi\}$, protected by the quantum phase noise $\phi_{\alpha}$. The contribution of short noise is averaged to zero. Since the electronic noise is not completely random and present in our measurement method, the noise will create statistical errors in the mean-value measurement of the coherent part of the multiplied signal. Note that the measurement method is also applied for large mean photon number coherent states as shown in Fig.2(d) and also for the mixture of stable and noise fields [14]. The aim of the postmeasurement method is to make sure that weak coherent state for quantum key distribution can provide coherent state bi-partite correlation, which can be used as a supplement for the decoy state BB84 and coherent state DPS-QKD. From the Fig.2(h), we obtain the product of the mean photon number fluxes for the $|\alpha|^{2}|\beta|^{2}$, where the $-\cos 2\left(\theta_{1}-\theta_{2}\right)=-1$ is maximum obtainable value for $\theta_{1}=\theta_{2}$. Fig. 2(i) shows that the multiplied signal is proportional to $-\cos 2\left(\theta_{1}-\theta_{2}\right)=1$ when the relative angle is set to $\theta_{1}-\theta_{2}=\pi / 2$. We are able to prepare four types of bi-partite correlations such as $\pm \cos 2\left(\theta_{1} \pm \theta_{2}\right)$ shared between two parties by using liner phase shifters on either beam 1 or beam 2. For practical quantum communication, Alice can keep the beam 1 and linear phase shifters as highlighted in the box in Fig.1, and send out the beam 2 to Bob. Since Alice can change the phases of beam 1 locally, her acts will change the coherent state bi-partite correlation function shared with Bob. Fig.3(a) shows that the normalized coherent state bi-partite correlation function $-\cos 2\left(\theta_{1}-\theta_{2}\right)$. For each data point, we take 10 shots of the multiplied signal and obtain the average mean-value. The error bar is mainly due to the electronic noises. The offset of the relative angle due to imperfection of quarter wave-plates has been corrected. For preparing the correlation function of $\cos 2\left(\theta_{1}+\theta_{2}\right)$ in Fig.3(b), we set the $\lambda / 4$ wave-plate in beam 1 to $-45^{\circ}$ so that the beat intensity $\mathcal{A}_{1}$ of Eq. 5 becomes $\mathcal{A}_{1} \propto-\cos \left(2 \theta_{1}-\left(\phi_{L O}-\phi_{\alpha}\right)\right)$. As for the correlation function of $\cos 2\left(\theta_{1}-\theta_{2}\right)$ in Fig. $3(\mathrm{c})$, we insert a $\lambda / 2$ plate at $0^{\circ}$ in beam 1 so that the minus sign of beat intensity $\mathcal{A}_{1}$ of Eq. 5 is changed to positive sign. Similarly, with the $\lambda / 2$ wave-plate at $0^{\circ}$ and the $\lambda / 4$ wave-plate at $-45^{\circ}$ in beam 1 , the beat signal $\mathcal{A}_{1}$ of Eq. 5 becomes $\cos \left(2 \theta_{1}-\left(\phi_{L O}-\phi_{\alpha}\right)\right)$. Thus, the correlation function of $-\cos 2\left(\theta_{1}+\theta_{2}\right)$ is obtained 


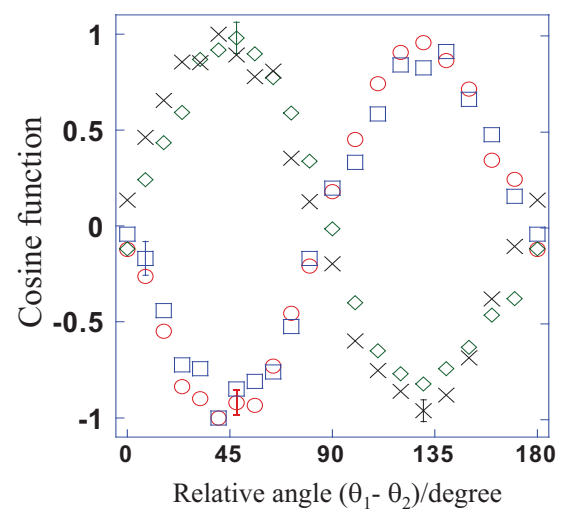

FIG. 3: The coherent state bi-partite correlations for $(\mathrm{a})(\mathrm{box})-\cos 2\left(\theta_{1}-\theta_{2}\right)$, (b)(diamond) $\cos 2\left(\theta_{1}+\theta_{2}\right)$, (c)(cross) $\cos 2\left(\theta_{1}-\theta_{2}\right)$, and (d)(circle) $-\cos 2\left(\theta_{1}+\theta_{2}\right)$.

as shown in Fig.3(d).

After we establish or choose one of coherent state bi-partite correlation functions between Alice and Bob, we implement bits correlations between them. To perform this measurement for the established correlation function of $-\cos 2\left(\theta_{1}-\theta_{2}\right)$, we ramp the PZT to obtain one period of interference signal. We reduce the average power of the weak LO field to $-39 \mathrm{dBm}$ and the average power of the weak coherent state to $-39 \mathrm{dBm}$. The output of the balanced homodyne beat intensity at the detector $\mathrm{A}$ is directly connected to a lock-inamplifier, where the reference frequency at $168 \mathrm{~Hz}$ is obtained from a function generator that drives the PZT. We measure quadrature phases of weak coherent state with the step size of $n \pi / 2$ ( $\mathrm{n}=$ integer) as shown in Fig.4(a)(solid line). Using the same lock-in reference phase in the lock-in-amplifier, we measure the quadrature phases of weak coherent state at detector $\mathrm{B}$ as shown in Fig.4(a)(dashed-line). We have observed the bits correlations between two parties for the shared correlation function of $-\cos 2\left(\theta_{1}-\theta_{2}\right)$ as shown in Fig.4 (a), where the positive (negative) quadrature signal is encoded as keys/bits '1' ('0'), respectively. By using the same lock-in reference phases, we observe bits correlations for other three types of correlation functions $-\cos 2\left(\theta_{1}+\theta_{2}\right), \cos 2\left(\theta_{1}+\theta_{2}\right)$, and $\cos 2\left(\theta_{1}-\theta_{2}\right)$ as shown in Fig.4(b), (c) and (d), respectively. In real practice, we can establish one of the CSBC for calibrating the lock-in reference phases at Alice and Bob.

We test the scheme by performing bits correlations between two parties over a distance of $10 \mathrm{~km}$ through a transmission fiber. We couple the beam 2 into the transmission fiber. A quarter wave plate and a half wave plate (not shown in Fig.1) are used at the output 


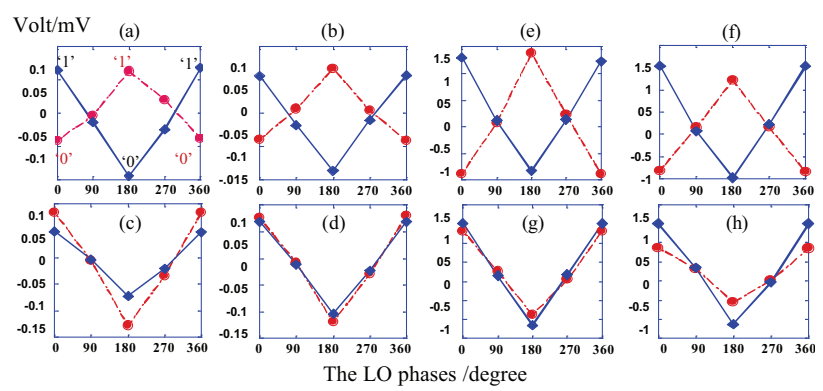

FIG. 4: Bits correlations for CSBC of ((a)and (e)) - $\cos 2\left(\theta_{1}-\theta_{2}\right) ;((\mathrm{b})$ and $(\mathrm{f}))-\cos 2\left(\theta_{1}+\theta_{2}\right) ;((\mathrm{c})$ and (g)) $\cos 2\left(\theta_{1}+\theta_{2}\right) ;((\mathrm{d})$ and $(\mathrm{h})) \cos 2\left(\theta_{1}-\theta 2\right)$, where (e), (f), (g) and (h) are with the $10 \mathrm{~km}$ fiber. The size of the data point is the error bar for the measurement.

of the transmission fiber to compensate the birefringence. Since there are losses in the couplings and the fiber, we use weak coherent state with average power of $-33 \mathrm{dBm}$ and weak local oscillator field with average power of $-33 \mathrm{dBm}$ before the homodyne detections. We have established four types of correlation functions and performed bits correlations for each shared correlation function between two parties as shown in Fig.4(e), (f), (g) and (h).

As a supplement to the DPF-QKG, the phase of the weak LO field can be randomly modulated as $\{0, \pi\}$ at certain frequency. Then, the bits/keys correlations can be realized based on the established CSBC shared by both parties. Since the established CSBC is normalized with the product of mean photon numbers $|\alpha|^{2}|\beta|^{2}$, photon number splitting attack can be detected by adding a weak LO beam in the decoy state BB84 protocol to check the CSBC shared between two parties. The security analysis of the scheme is out of the scope of this paper.

Intrinsic correlations of coherent light field have been utilized to implement entanglement [15], Grover search algorithm [16, 17], quantum lithography [18], factoring number [19] and quantum walk [20] through different well-designed interference measurement methods. Intrinsic correlations of coherent states do not exhibit non-locality as two-photon source. The realization of intrinsic quantum correlation of weak coherent state by using the measurement method is a first step toward linear-optics quantum computing with weak light fields and single-photon source.

The authors would like to acknowledge that this paper is prepared under the support of the start-up fund from Department of Physics, Michigan Technological University. Erin Scanlon and Viktor Bollen would like to acknowledge that the support of SURF to carry 
out the experiment under supervision of Kim Fook Lee.

* kflee@mtu.edu

[1] A. K. Ekert, Phys. Rev. Lett. 67, 661 (1991).

[2] K. Inoue, E. Waks, and Y. Yamamoto, Phys. Rev. Lett. 89, 037902 (2002); Phys. Rev. A 68, $022317(2003)$

[3] K. Wen, K. Tamaki, and Y. Yamamoto, Phys. Rev. Lett. 103, 170503 (2009).

[4] H. K. Lo, X. Ma, and K. Chen, Phys. Rev. Lett. 94, 230504 (2005).

[5] X. B. Wang, Phys. Rev. Lett. 94, 230503 (2005).

[6] Y. Zhao, B. Qi, X. Ma, H. K. Lo, and L. Qian, Phys. Rev. Lett. 96, 070502 (2006).

[7] D. Rosenberg et al, Phys. Rev. Lett. 98, 010503 (2007).

[8] C. Z. Peng, J. Zhang, and D. Yang, Phys. Rev. Lett. 98, 010505 (2007).

[9] T. Schmitt-Manderbach et al, Phys. Rev. Lett. 98, 010504 (2007).

[10] G. A. Barbosa, E. Corndorf, P. Kumar, and H. Yuen, Phys. Rev. Lett. 90, 227901 (2003).

[11] A. Kuzmich, I. A. Walmsley, and L. Mandel, Phys. Rev. Lett. 85, 1349 (2000); Phys. Rev. A 64, 063804 (2001).

[12] Z. Y. Ou, C. K. Hong, and L. Mandel, Opt. Commun. 63, 118 (1987).

[13] W. P. Schleich, Quantum Optics in Phase-Space, Beam splitter transformation in Chapter 13, 1 edition, Wiley-VCH, (2001).

[14] K. F. Lee, Opt. Lett. 34, 1099 (2009).

[15] K. F. Lee, and J. E. Thomas, Phys. Rev. A 69, 052311 (2004); Phys. Rev. Lett. 88, 097902 (2002).

[16] S. Lloyd, Phys. Rev. A 61, 010301 (2000).

[17] N. Bhattacharya, H. B. Van Linden, V. den Heuvell, and R. J. Spreeuw, Phys. Rev. A 63, $062302(2001)$.

[18] P. R. Hemmer, A. Muthukrishnan, M. O. Scully, and M. S. Zubairy, Phys. Rev. Lett. 96, $163603(2006)$.

[19] D. Bigourd, B. Chatel, W. P. Schleich, and B. Girard, Phys. Rev. Lett. 100, 030202 (2008).

[20] H. B. Perets et al, Phys. Rev. Lett. 100, 170506 (2008). 